\title{
Frequent silencing of the candidate tumor suppressor TRIM58 by promoter methylation in early-stage lung adenocarcinoma
}

\author{
Koichiro Kajiura ${ }^{1,2, *}$, Kiyoshi Masuda ${ }^{1, *}$, Takuya Naruto $^{1}$, Tomohiro Kohmoto ${ }^{1}$, \\ Miki Watabnabe ${ }^{1}$, Mitsuhiro Tsuboi ${ }^{2}$, Hiromitsu Takizawa ${ }^{2}$, Kazuya Kondo ${ }^{3}$, Akira \\ Tangoku ${ }^{2}$, Issei Imoto ${ }^{1}$ \\ ${ }^{1}$ Department of Human Genetics, Graduate School of Biomedical Sciences, Tokushima University, Tokushima, Japan \\ ${ }^{2}$ Department of Thoracic, Endocrine and Oncological Surgery, Graduate School of Biomedical Sciences, Tokushima University, \\ Tokushima, Japan \\ ${ }^{3}$ Department of Oncological Medical Services, Graduate School of Biomedical Sciences, Tokushima University, Tokushima, \\ Japan \\ *These authors contributed equally to this work
}

Correspondence to: Issei Imoto, email: issehgen@tokushima-u.ac.jp

Keywords: TRIM58, early-stage lung adenocarcinoma, tumor suppressor gene, methylation, smoking status

Received: August 26, 2016

Accepted: November 22, 2016

Published: December 01, 2016

\section{ABSTRACT}

In this study, we aimed to identify novel drivers that would be epigenetically altered through aberrant methylation in early-stage lung adenocarcinoma (LADC), regardless of the presence or absence of tobacco smoking-induced epigenetic field defects. Through genome-wide screening for aberrantly methylated CpG islands (CGIs) in 12 clinically uniform, stage-I LADC cases affecting six non-smokers and six smokers, we identified candidate tumor-suppressor genes (TSGs) inactivated by hypermethylation. Through systematic expression analyses of those candidates in panels of additional tumor samples and cell lines treated or not treated with 5-aza-deoxycitidine followed by validation analyses of cancer-specific silencing by CGI hypermethylation using a public database, we identified TRIM58 as the most prominent candidate for TSG. TRIM58 was robustly silenced by hypermethylation even in early-stage primary LADC, and the restoration of TRIM58 expression in LADC cell lines inhibited cell growth in vitro and in vivo in anchorage-dependent and -independent manners. Our findings suggest that aberrant inactivation of TRIM58 consequent to CGI hypermethylation might stimulate the early carcinogenesis of LADC regardless of smoking status; furthermore, TRIM58 methylation might be a possible early diagnostic and epigenetic therapeutic target in LADC.

\section{INTRODUCTION}

Lung adenocarcinoma (LADC) is the most predominant histologic subtype of lung cancer and one of the leading causes of cancer-related death worldwide $[1,2]$. Although major improvements in treatment strategies have increased the short-term survival of patients with LADC, the impacts on long-term survival have remained modest [3]. Both late diagnosis and the scarcity of therapies that effectively achieve durable responses are important factors associated with poor outcomes. A better understanding of the molecular pathogenesis of LADC is needed to identify biomarkers that could be useful for early detection and to develop novel targeted therapies.

Aberrant promoter $\mathrm{CpG}$ island (CGI) methylation of tumor suppressor genes (TSGs) has been established as a common epigenetic mechanism underlying the pathogenesis of human cancers, including LADC $[4,5]$. DNA methylation is a stable modification that often occurs at an early stage of carcinogenesis; as a result, such events are sensitive and specific biomarkers, even in tumor samples contaminated by non-tumorous cells as well as various biological fluids used for liquid biopsies. In LADC, distinct interactions between genetic and epigenetic changes were found to contribute differently 
to carcinogenesis in smokers (S) vs. never smokers (NS) $[6,7]$. However, few genes that are altered by aberrant DNA methylation irrespective of etiology (particularly smoking status) and contribute to early LADC carcinogenesis have been identified.

In the present study, we performed a systematic, genome-wide screening of aberrantly methylated CGIs in stage-I LADCs from both S and NS to identify genes commonly dysregulated via aberrant DNA methylation in early-stage LADC, regardless of smoking status. To this end, we applied a series of criteria that targeted novel genes frequently silenced in LADC tumors and cell lines (possibly through hypermethylation) to prioritize the selection of top candidates for validation. TRIM58, a subsequently identified candidate, showed frequent CGI hypermethylation and its inverse relationship with gene expression in a larger set of LADC tumors and cell lines, followed by validation using a data set obtained from The Cancer Genome Atlas (TCGA). In addition, our functional analyses demonstrated the ubiquitin-ligase activity-dependent growth suppressive effect of TRIM58 on LADC cells both in vitro and in vivo, suggesting that this gene exerts TSG during the initiation and/or early progression stages of LADC.

\section{RESULTS}

\section{Screening of aberrantly methylated and silenced genes from the early stage of LADC}

We initially screened tumors and paired nontumorous tissues obtained from 12 male patients with stage-I LADC (6 S, 6 NS; Table 1) using an Illumina HumanMethylation450K BeadChip to identify differentially methylated CGIs in a genome-wide manner (Figure 1A). Because the number of hypermethylated CGIs was much larger than that of hypomethylated CGIs (Figure 1B), we focused on the former group. A total of 113 CGIs were identified as differentially hypermethylated CGIs, with a false discovery rate (FDR) of $<0.05$ and $\beta$ difference (tumor - non-tumorous tissue) of $>0.25$.

Next, we determined the mRNA expression of 9 genes associated with the top 10 CGIs (Table 2) in commercially available normal lung, panels of 11 stage-I or -II LADC cases (Supplementary Table S1), and 14 LADC cell lines via quantitative reverse-transcription polymerase chain reaction (qRT-PCR). Differential hypermethylation in tumors of those genes except MEIS2 was validated using 29 paired cases of a TCGA data set (Supplementary Table S2). Three genes (DDX25, FEZF2, and C12orf42) that exhibited no or very low expression in commerciallyavailable normal lung and non-tumorous lung tissues (data not shown) as well as 58 non-tumorous lung tissues from the TCGA data set (Supplementary Figure S1). Three genes (ZNF577, MEIS2, and PTPRN2) were infrequently downregulated in LADC tumors relative to paired non-tumorous tissues and/or LADC cell lines compared with normal lung (Supplementary Figures S2 and S3). The TCGA data set validated significant but small reductions in the expression levels of these genes in tumors relative to non-tumorous tissues (Supplementary Table S2).

Finally, we conducted pharmacological re-expression experiments in LADC cell lines treated with vehicle, 5-aza2'-deoxycitidine (5-aza-dC) and/or trichostatin A (TSA). Only TRIM58 restoration was effectively ( $\log _{2}$ (fold change) $>2$ ) and consistently (3/3 lines) observed throughout 5-aza$\mathrm{dC}$ treatment (Figure 1E and Supplementary Figure S4A). In addition, the effect of 5-aza-dC treatment on TRIM58 expression was not observed in TRIM58-expressing cell lines (Supplementary Figure S4B). Accordingly, TRIM58 was identified as the most likely candidate for DNA methylation-induced silencing from the early stages of LADC, irrespective of smoking status. Decreased TRIM58 mRNA expression in tumors relative to paired nontumorous tissues was consistently observed even after the inclusion of 27 additional cases with various stages and smoking statuses (Figure 1C).

\section{Expression and CGI methylation status at the TRIM58 promoter in LADC}

Using methylation array data of 12 stage-I cases, we analyzed details regarding the methylation status of CpG sites around TRIM58. CpG sites within the CGI exhibited low levels of methylation $(\beta$ value $=0.1-0.2)$ in non-tumorous tissues and significantly higher levels in paired tumors (Figure 2A), irrespective of the patient's smoking status (Supplementary Figure S5A). Identical results were obtained in the TCGA data set $(n=29$, Supplementary Figure S5B).

Because almost all clinical cases exhibited decreased TRIM58 expression, the correlation between the CGI methylation status and TRIM58 expression status was first assessed in LADC cell lines (Figures 1D and 2B). Quantitative pyrosequencing analysis of five target $\mathrm{CpG}$ sites within the TRIM58 CGI revealed low methylation levels in most $\mathrm{CpG}$ sites in all three LADC cell lines with higher-than-normal TRIM58 expression levels, whereas cell lines with very low TRIM58 expression (except RERF-LC-KJ) exhibited high levels of methylation, particularly at cg20429172 (target III) and cg26157385 (target IV). In three 5-aza-dC-treated cell lines with restored TRIM58 mRNA expression, the methylation levels decreased to some extent at all target sites.

The methylation statuses of $\mathrm{CpG}$ sites within the TRIM58 CGI around cg20429172 and cg26157385 were confirmed by bisulfite sequencing in TRIM58-expressing (RERF-LC-MS) and TRIM58-nonexpressing (NCI-H358) cells treated or not with 5-aza-dC (Figure 2C). A promoter reporter assay using various DNA fragments overlapped with the CGI in both TRIM58-expressing and nonexpressing cell lines demonstrated that only fragments 
Table 1: Clinicopathological characteristics of patients with LADC analyzed by Infinium HumanMethylation450K BeadChips

\begin{tabular}{lccccccc}
\hline \multirow{2}{*}{ ID } & \multirow{2}{*}{$\begin{array}{c}\text { Age } \\
(\mathbf{y r s})\end{array}$} & Gender & Smoking status & \multirow{2}{*}{ Pack-years } & \multicolumn{3}{c}{ Tumor } \\
\cline { 6 - 8 } & & & & & TNM classification & Stage & Size $(\mathbf{m m})$ \\
\hline S1 & 68 & Male & Smoker & 1000 & pT1aN0M0 & Ia & $13 \times 11$ \\
S2 & 80 & Male & Smoker & 1200 & pT1aN0M0 & Ia & $14 \times 11$ \\
S3 & 60 & Male & Smoker & 1020 & pT2aN0M0 & Ib & $17 \times 11$ \\
S4 & 59 & Male & Smoker & 1600 & pT1aN0M0 & Ia & $13 \times 10$ \\
S5 & 74 & Male & Smoker & 2400 & pT1aN0M0 & Ia & $12 \times 11$ \\
S6 & 75 & Male & Smoker & 1000 & pT1aN0M0 & Ia & $25 \times 20$ \\
NS1 & 77 & Male & Never smoker & 0 & pT1aN0M0 & Ia & $20 \times 13$ \\
NS594 & 74 & Male & Never smoker & 0 & pT1aN0M0 & Ia & $19 \times 17$ \\
NS3 & 57 & Male & Never smoker & 0 & pT1aN0M0 & Ia & $15 \times 15$ \\
NS4 & 73 & Male & Never smoker & 0 & pT2aN0M0 & Ib & $42 \times 19$ \\
NS5 & 51 & Male & Never smoker & 0 & pT1aN0M0 & Ia & $14 \times 9$ \\
NS6 & 68 & Male & Never smoker & 0 & pT1aN0M0 & Ia & $13 \times 11$ \\
\hline
\end{tabular}

2 and 4 containing cg20429172 and cg26157385 exhibited significant promoter activity regardless of the endogenous TRIM58 expression status (Figure 2D), suggesting that the methylation of cis-regulatory $\mathrm{CpG}$ sites within fragment 4 most likely determines, at least in part, the TRIM 58 mRNA expression level.

Using normalized RNA-seq gene expression data and HumanMethylation450K BeadChip data of 17 LADC tumors and paired non-tumorous tissues from the TCGA data set, we confirmed an inverse correlation between the TRIM58 expression and methylation levels at five pyrosequencing target sites (Supplementary Figure S5C). At those sites, particularly cg20429172 and $\operatorname{cg} 26157385$, mutually exclusive plotting of the expression and methylation levels was observed between tumors and nontumorous tissues. In a larger set of our LADC cases (46 cases), pyrosequencing demonstrated the smoking statusindependent, consistent tumor-specific hypermethylation of cg26157385 (Figure 3A).

To evaluate the TRIM58 protein expression status in LADC tumors and paired non-tumorous lung tissues, immunohistochemical staining (IHC) was performed (Figure 3B). In non-tumorous lung tissues, cytoplasmic TRIM58 staining was observed in alveolar epithelial cells, especially type II cells, whereas almost no TRIM58 staining was observed in LADC tumor cells, irrespective of the smoking status and tumor stage.

\section{TRIM58 overexpression suppresses LADC cell proliferation and tumor formation in vitro and in vivo}

To investigate the biological significance of TRIM58 in the carcinogenesis of LADC, TRIM58 expression was transiently or stably restored in LADC cells lacking its endogenous expression. Because TRIM58 was silenced in most LADC cases even at early stages, we evaluated proliferation and tumor formation in those cells both in vitro and in vivo. TRIM58 is a member of the tripartite motif (TRIM)-containing protein family, which mainly comprises E3 ubiquitin (Ub) ligases containing a RINGfinger domain with E3 Ub ligase activity. To determine whether the effects of TRIM58 depend on its Ub ligase activity, we simultaneously assessed a wild-type TRIM58 (TRIM58-WT) and a mutated form that lacked polyubiquitination activity consequent to a switch of histidine 33 to alanine within the RING-finger domain (TRIM58Mut, Figure 4A) with the control counterparts (empty vector transfectants).

In colony-formation assays involving transiently transfected cells, fewer colonies were produced by TRIM58-WT-transfected LADC cell lines when compared to control and TRIM58-Mut-transfected cells (Figure 4B). In an in vitro proliferation assay of stably transfected cells, cells expressing exogenous TRIM58-WT protein (predominant cytoplasmic location) grew significantly more slowly than mock-transfected controls and TRIM58Mut-expressing cells (Figure 5A). Notably, p21 expression was pronounced only in TRIM58-WT-overexpressing cells, whereas a minimal effect of TRIM58-WT overexpression on PARP cleavage was observed in A549 cells and no effect on PARP cleavage was observed in HCC827 cells (Figure 5B). In a flow-assisted cell sorting (FACS) analysis in A549 cells to examine the mode of action of TRIM58 within the cell cycle, we observed an accumulation of cells in $\mathrm{G}_{0}-\mathrm{G}_{1}$ phase and decreases in cells in the $\mathrm{S}$ and $\mathrm{G}_{2}-\mathrm{M}$ phase without an increase in sub- $\mathrm{G}_{1}$ phase cells among TRIM58-WT-transfected cells relative 
A

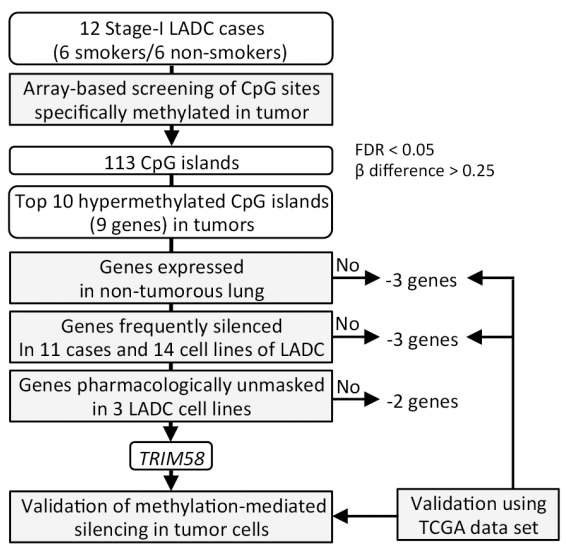

B

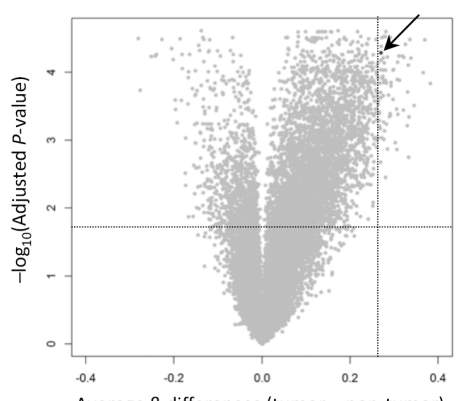

Average $\beta$ differences (tumor - non-tumor)

C

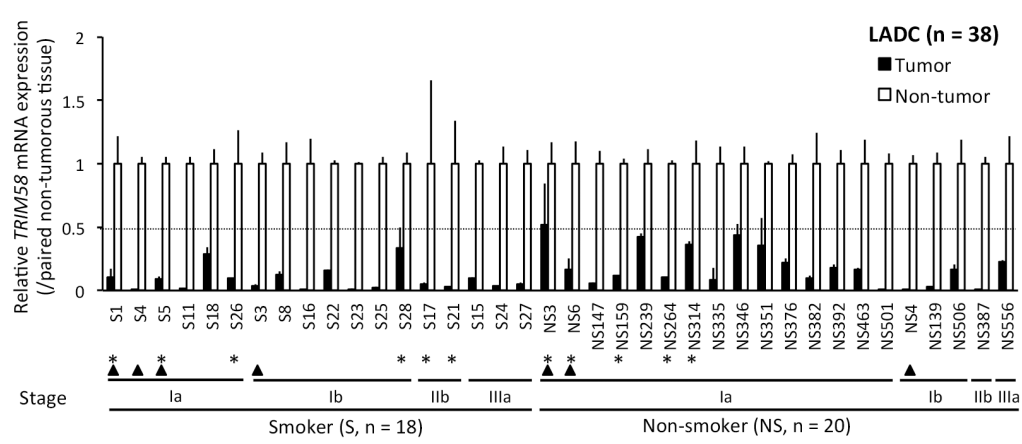

D

$E$
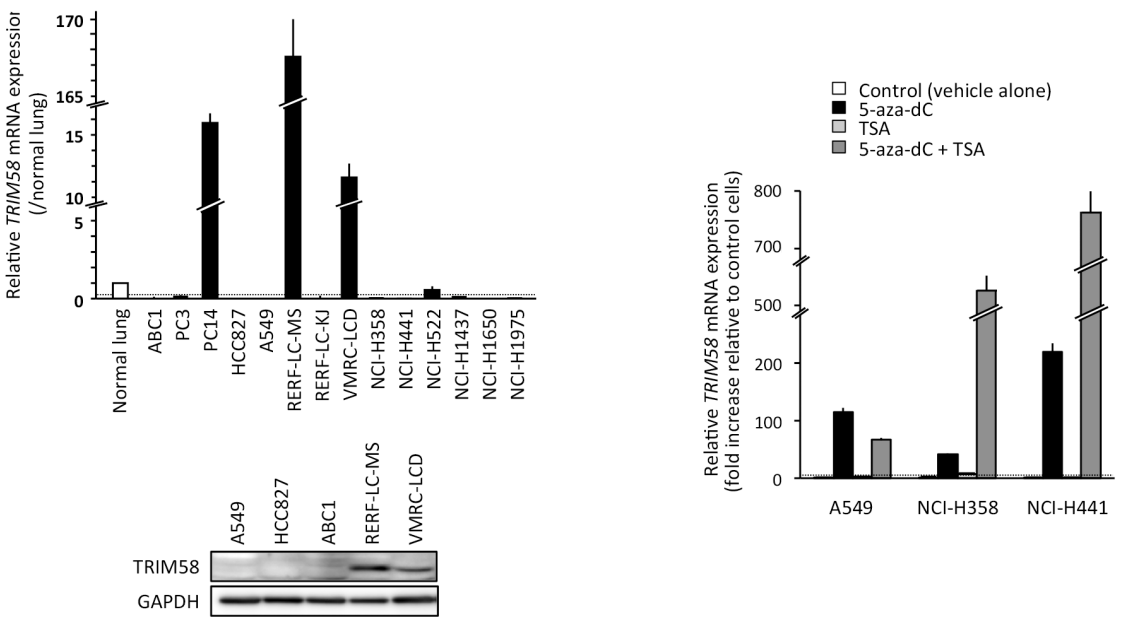

Figure 1: TRIM58 was identified as a candidate in a screening of genes silenced through hypermethylation of the CpG islands (CGI) in lung adenocarcinomas (LADCs) from an early stage, regardless of patient smoking status. (A) Flow chart indicating the study design, which aimed to identify candidate tumor suppressor genes using experimental and bioinformatic filters. (B) Volcano plot of the differential CGI methylation statuses of 12 stage-I LADC tumors and paired non-tumorous tissues. The $\mathrm{x}$-axis indicates the average difference in $\beta$-value (methylation level), and the y-axis indicates the- $\log 10$ of the adjusted $P$-value for each CGI. The arrow indicates the CGI around TRIM58. (C) Relative expression (fold change) of TRIM58 mRNA in tumor tissues vs. paired non-tumorous tissues in a panel of LADC cases ( $n=38$ including 18 smokers [S] and 20 non-smokers [NS]; Supplementary Table S1) evaluated by qRTPCR. Expression data were normalized to GAPDH (means \pm standard deviations [SD] of triplicate experiments). Asterisks indicate 11 cases used to screen candidate gene expression (Supplementary Figure S2); arrowheads indicate cases used for array-based methylation screening (see Table 1). Dotted line indicates 0.5. (D) Expression levels of TRIM58 mRNA (upper) and protein (lower) in LADC cell lines. Upper, relative expression levels of TRIM58 mRNA in 14 LADC cell lines vs. normal lung tissues; expression was evaluated using qRT-PCR and normalized to GAPDH. Dotted line indicates 0.2. Lower, TRIM58 protein expression in five cell lines was determined by western blotting with a TRIM58-specific antibody. (E) Effect of treatment with 5-aza-dC and/or TSA on TRIM58 mRNA expression in three LADC cell lines. The expression levels of TRIM $58 \mathrm{mRNA}$, which were evaluated by qRT-PCR and normalized to GAPDH, are shown relative to those of vehicle-treated control cells (means \pm SDs of triplicate experiments). Dotted line indicates four (fold change). 
A

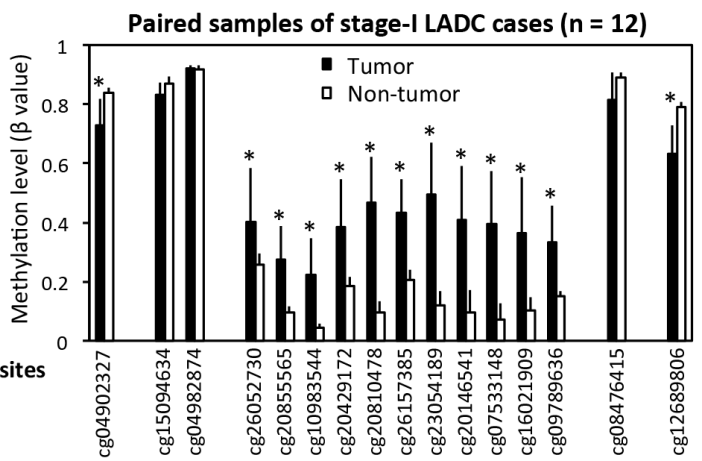

Pyrosequencing

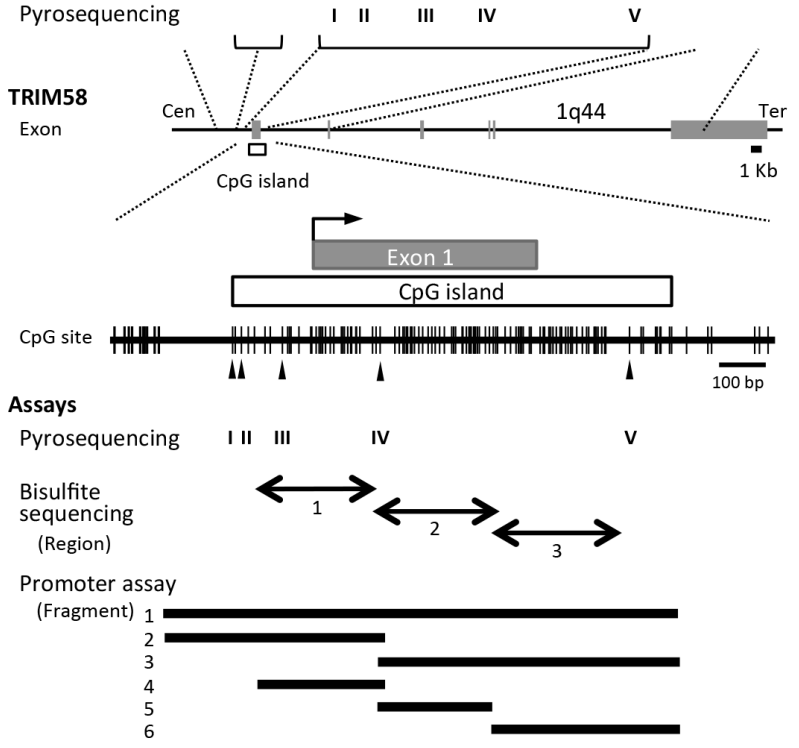

B
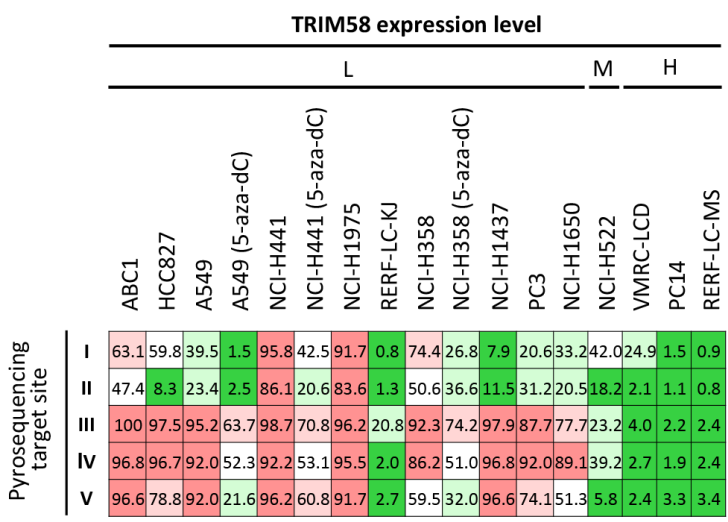

\begin{tabular}{c|c|c|c|c|c|}
$\sim 20$ & $\sim 40$ & $\sim 60$ & $\sim 80$ & $80^{\sim}$ & $(\%)$
\end{tabular}

C

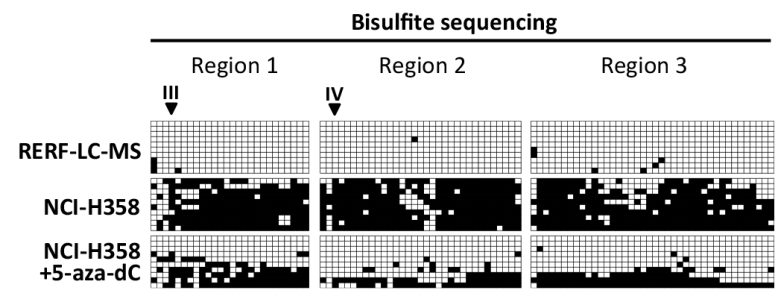

D
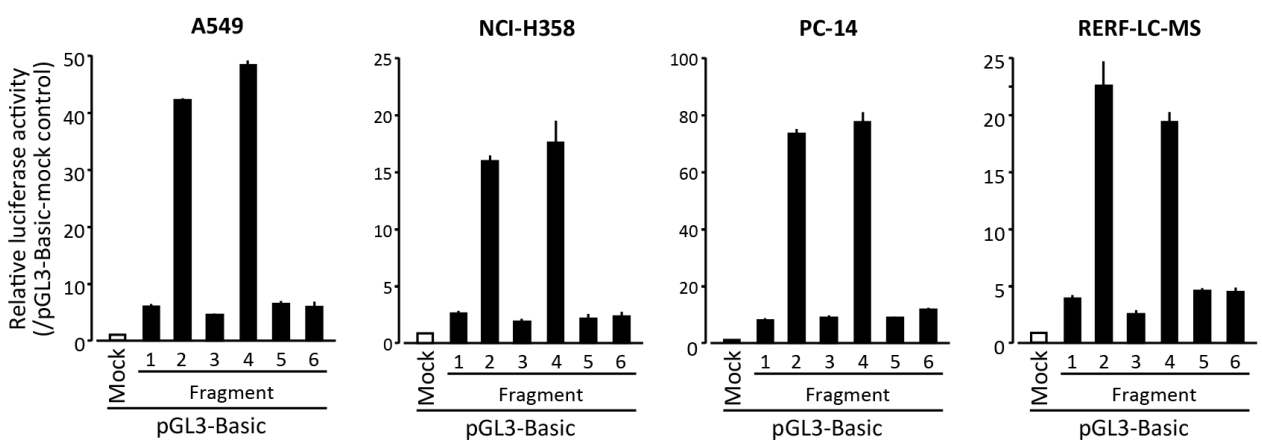

Figure 2: Correlation of CpG site methylation with the TRIM58 expression status in lung adenocarcinoma (LADC) cell lines. (A) A schematic diagram of the TRIM58 gene structure and $\mathrm{CpG}$ sites around exon 1 (middle), the average $\beta$-value (methylation level) of each $\mathrm{CpG}$ site targeted in the array-based methylation experiment involving $12 \mathrm{LADC}$ cases (upper), and the CpG sites around exon 1 and the $\mathrm{CpG}$ island (CGI) with $\mathrm{CpG}$ sites targeted by pyrosequencing (arrowhead, $\mathrm{I}-\mathrm{V}$ ), as well as the regions analyzed via bisulfite sequencing (closed arrows, regions 1-3) and promoter assays (horizontal bar, segments $1-6)$ ). ${ }^{*} P<0.05$ vs. paired non-tumorous tissue. (B) Average DNA methylation values, shown as percentages from quantitative pyrosequencing to analyze five target sites in 14 cell lines, including three cell lines treated with 5-aza-dC (triplicate experiments). Results were classified into five grades according to $20 \%$ quintiles. Cells were divided into three groups according to the expression of TRIM58 mRNA relative to the normal lung: $\mathrm{H}, \geq$ normal lung; $\mathrm{M}$, $<$ normal lung but $\geq 20 \%$ of the normal lung; $\mathrm{L},<20 \%$ of the normal lung. (C) Bisulfite sequencing of part of the TRIM58 CGI (see Figure 2A) in RERF-LC-MS, NCI-H358, and 5-aza-dC-treated NCI-H358 cells. Open and filled squares represent unmethylated and methylated $\mathrm{CpG}$ sites, respectively, and each row represents the results for a single clone. Arrowheads, target sites for pyrosequencing. (D) Luciferase assays involving pGL3 constructs containing various fragments around the TRIM58 CGI (see Figure 2A) in TRIM58 nonexpressing and expressing LADC cell lines (see Figure 1D). Values are expressed as the fold activation relative to pGL3-mock transfected cells (means \pm standard deviations of three independent experiments). 
Table 2: Top $10 \mathrm{CpG}$ islands significantly hypermethylated in tumors compared to paired nontumorous tissues of 12 stage-I LADC cases

\begin{tabular}{|c|c|c|c|c|c|c|c|c|c|}
\hline \multirow[b]{2}{*}{ Rank } & \multirow[b]{2}{*}{ CpG island } & \multicolumn{2}{|c|}{$\begin{array}{l}\text { Methylation status of } \\
\text { CpG island }\end{array}$} & \multicolumn{2}{|c|}{$\begin{array}{l}\text { CpG island-related } \\
\text { RefSeq gene }{ }^{\mathrm{a}}\end{array}$} & \multicolumn{4}{|c|}{ Expression status of CpG island-related gene ${ }^{\mathrm{b}}$} \\
\hline & & $\begin{array}{l}\text { Adjusted } \\
P \text {-value }\end{array}$ & $\beta$-difference ${ }^{d}$ & $\begin{array}{l}\text { Gene } \\
\text { name }\end{array}$ & $\begin{array}{c}\text { Location } \\
\text { of CpG } \\
\text { islande }\end{array}$ & $\begin{array}{c}\text { Expression } \\
\text { in normal } \\
\text { lung }^{\mathrm{f}}\end{array}$ & $\begin{array}{c}\text { Low } \\
\text { expression } \\
\text { in tumors of } \\
\text { early LADC } \\
\text { cases }^{\mathrm{g}}\end{array}$ & $\begin{array}{c}\text { Low } \\
\text { expression } \\
\text { in LADC } \\
\text { cell lines }^{\mathrm{h}}\end{array}$ & $\begin{array}{c}\text { Restration of } \\
\text { expression in } \\
\text { 5-aza-dC-treated } \\
\text { LADC cell lines }\end{array}$ \\
\hline 1 & $\begin{array}{l}\text { chr7:153583317- } \\
153585666\end{array}$ & $2.92 \mathrm{E}-05$ & 0.2776 & $D P P 6$ & Exon 1 & Yes & 8/11 & $14 / 14$ & $1 / 3$ \\
\hline 2 & $\begin{array}{l}\text { chr11:125774292- } \\
125774584\end{array}$ & $3.04 \mathrm{E}-05$ & 0.2719 & $D D X 25$ & Exon 1 & Very low & - & - & - \\
\hline 3 & $\begin{array}{l}\text { chr19:52390841- } \\
52391368\end{array}$ & $3.04 \mathrm{E}-05$ & 0.2915 & ZNF577 & Exon 1 & Yes & $6 / 11$ & $10 / 14$ & $0 / 3$ \\
\hline 4 & $\begin{array}{l}\text { chr3:62355315- } \\
62355534\end{array}$ & $3.24 \mathrm{E}-05$ & 0.2589 & $F E Z F 2$ & Gene body & Very low & - & - & - \\
\hline 5 & $\begin{array}{l}\text { chr1:156863415- } \\
156863711\end{array}$ & $3.26 \mathrm{E}-05$ & 0.3692 & PEAR1 & Exon 1 & Yes & $5 / 11$ & $14 / 14$ & $0 / 3$ \\
\hline 6 & $\begin{array}{l}\text { chr15:37390175- } \\
37390380\end{array}$ & $3.81 \mathrm{E}-05$ & 0.3249 & MEIS2 & Gene body & Yes & $4 / 11$ & $3 / 14$ & $1 / 3$ \\
\hline 7 & $\begin{array}{l}\text { chr1:248020330- } \\
248021252\end{array}$ & $5.10 \mathrm{E}-05$ & 0.2703 & TRIM58 & Exon 1 & Yes & $10 / 11(36 / 37)$ & $10 / 14$ & $3 / 3$ \\
\hline 8 & $\begin{array}{l}\text { chr3:62362610- } \\
62363082\end{array}$ & $5.44 \mathrm{E}-05$ & 0.2521 & $F E Z F 2$ & Upstream & - & - & - & - \\
\hline 9 & $\begin{array}{l}\text { chr12:103696090- } \\
103696418\end{array}$ & $5.70 \mathrm{E}-05$ & 0.3189 & C12orf42 & Gene body & Very low & - & - & - \\
\hline 10 & $\begin{array}{l}\text { chr7:158110569- } \\
158110881\end{array}$ & $6.58 \mathrm{E}-05$ & 0.2702 & PTPRN2 & Gene body & Yes & $3 / 11$ & $7 / 14$ & $2 / 3$ \\
\hline
\end{tabular}

${ }^{a}$ RefSeq gene (http://www.ncbi.nlm.nih.gov/refseq/) located on or around each $\mathrm{CpG}$ island was selected as CpG island-related gene.

bexpression status was evaluated in 9 genes, because two $\mathrm{CpG}$ islands located on one gene (FEZF2).

${ }^{c}$ Differences between methylation levels ( $\beta$-values) of $\mathrm{CpG}$ islands in tumors and paired non-tumorous tissues were assessed by paired $t$-test. $P$-values were adjusted with the Benjamini-Hochberg correction (False discovery rate, FDR). CpG islands were sorted by adjusted $P$-value.

${ }^{\mathrm{d}} \beta$-differences (differential methylation levels) represent the average of [( $\beta$-value of tumorous tissue) - ( $\beta$-value of paired non-tumorous tissue)] in 12 stage-I LADC cases.

eExon 1, CpG island locates within or contains exon 1 of gene; gene body, $\mathrm{CpG}$ island locates within or around exons other than exon 1 or introns; upstream, CpG island locates upstream of exon 1.

fVery low, expression level was undetermined by qRT-PCR in normal lung tissue (Clontech) and non-tumorous lung tissues in 11 cases with LADC.

${ }^{g}$ Low expression, $>50 \%$ reduction of relative gene expression level in tumors compared with paired non-tumorous tissues of early LADC cases. The boldface shows frequently ( $\geq 6 / 11$ cases) silenced genes.

${ }^{h}$ Low expression, $>80 \%$ reduction of relative gene expression level in cell lines compared with the normal lung tissue (Clontech). The boldface shows frequently ( $\geq 8 / 14$ cases) silenced genes.

${ }^{i}$ Restoration of expression, a $>4$-fold ( $\log _{2}>2$ fold) increase in mRNA expression in 5-aza-dC-treated cells compared to paired vehicle-treated cells (control). The boldface shows frequently $(\geq 2 / 3$ cell lines) restored genes.

to mock-transfected or TRIM58-Mut-transfected cells (Figure 5C). Similar results were obtained in HCC827 cells (Supplementary Figure S6), suggesting that wildtype TRIM58 contributes mainly to the arrest of LADC cells at the $\mathrm{G}_{1}-\mathrm{S}$ checkpoint probably through its Ub ligase activity. In addition, anchorage-independent in vitro 3D cell proliferation, determined via spheroid formation in low-attachment wells (Figure 5D) and colony formation in soft agar (Figure 5E), was also inhibited in TRIM58WT-transfected A549 cells relative to mock-transfected or TRIM58-Mut-transfected counterparts.

The ability of TRIM58 to suppress tumor formation in vivo was investigated using tumor xenograft experiments in which subcutaneous tumor growth was compared among severe combined immunodeficient (SCID) mice transplanted with different stable A549 transfectants. Restored expression of TRIM58-WT in A549 cells was associated with a reduced tumor volume and weight in vivo (Figure 6A, 6B, and Supplementary Figure S7). This outcome was attributed to a decrease in anchorage-dependent and/or -independent cell proliferation, as demonstrated by reduced Ki-67 positivity in TRIM58-WT-transfected cells relative to mocktransfected or TRIM58-Mut-transfected cells in resected tumors (Figure 6C).

Very recently, TRIM58 was found to facilitate erythroblast enucleation by interfering with the established ability of dynein to regulate intracellular 
nuclear positioning and/or microtubule structures during erythropoiesis [8]. However, the molecular mechanisms underlying the tumor-suppressive function of TRIM58 remain unknown and might be different from those involved in erythropoiesis. Indeed, our preliminary analysis demonstrated a lack of significant changes in the protein expression levels of dynein heavy chain 1 (DHC1) and intermediate chains (DIC), which were reported to be possible substrates of TRIM58 in proteasome-dependent degradation [8] between TRIM58-WT-overexpressing cells and mock-transfected or TRIM58-Mut-transfected counterparts (Supplementary Figure S8). To better elucidate the molecular mechanisms of tumor suppression by TRIM58, we performed microarray analysis to determine the effects of stable TRIM58 overexpression on the A549 cell transcriptome. We identified 1,889

A

Paired samples of LADC cases $(n=46)$
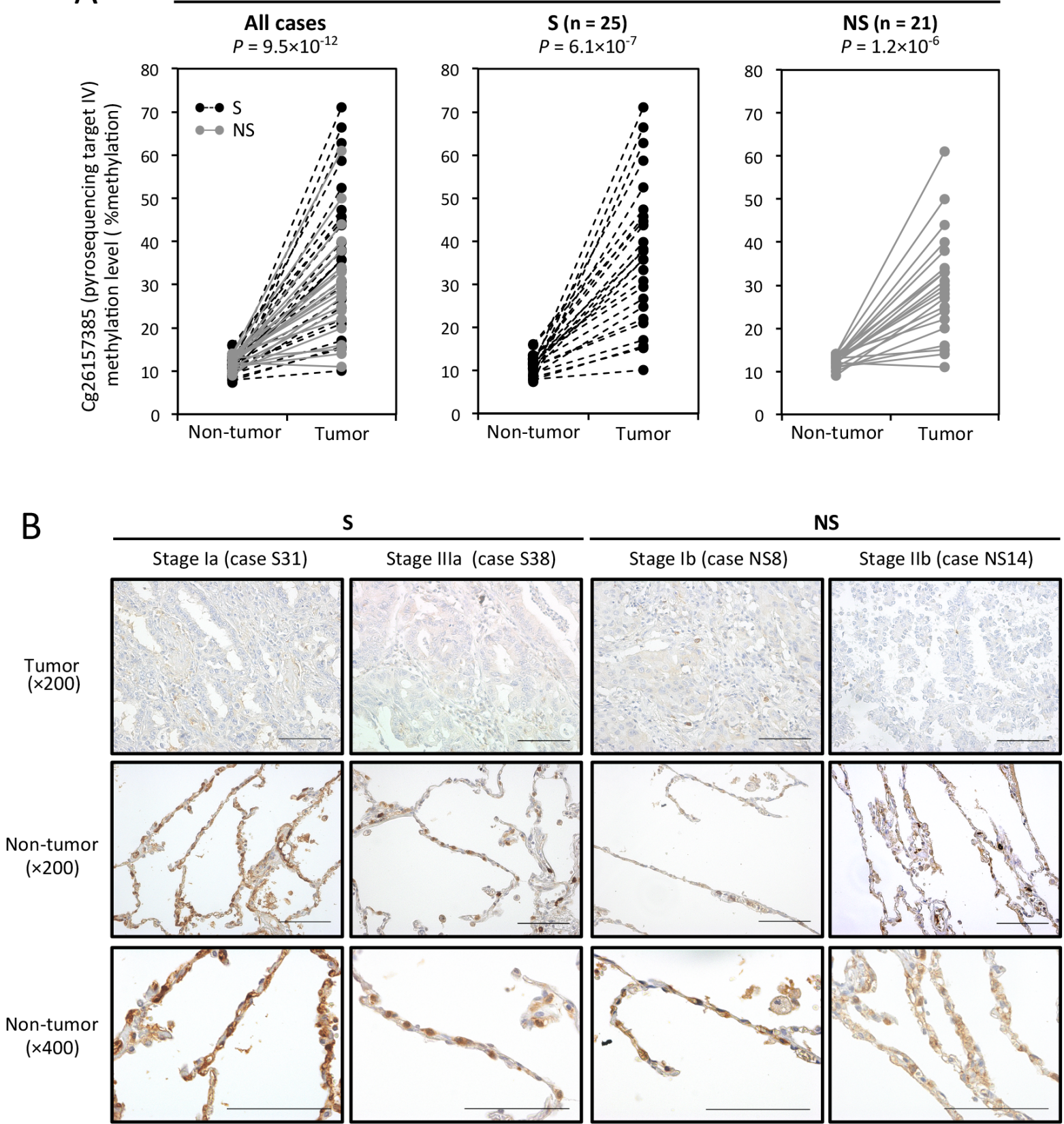

Figure 3: CpG site methylation and TRIM58 expression status in primary lung adenocarcinoma (LADC) tumors and paired non-tumorous tissues. (A) Average DNA methylation values (percentages) of cg26157385 (target IV, see Figure 2A) in 46 LADC tumors and paired non-tumorous tissues from 25 smokers (S) and 21 nonsmokers (NS), as determined by quantitative pyrosequencing. Samples from the same patient are linked with dotted or straight lines. (B) Representative images of immunohistochemical staining for TRIM58 in LADC tumors and adjacent non-tumorous lung tissues. S and NS indicate smokers and nonsmokers, respectively. Scale bars, $200 \mu \mathrm{m}$. 

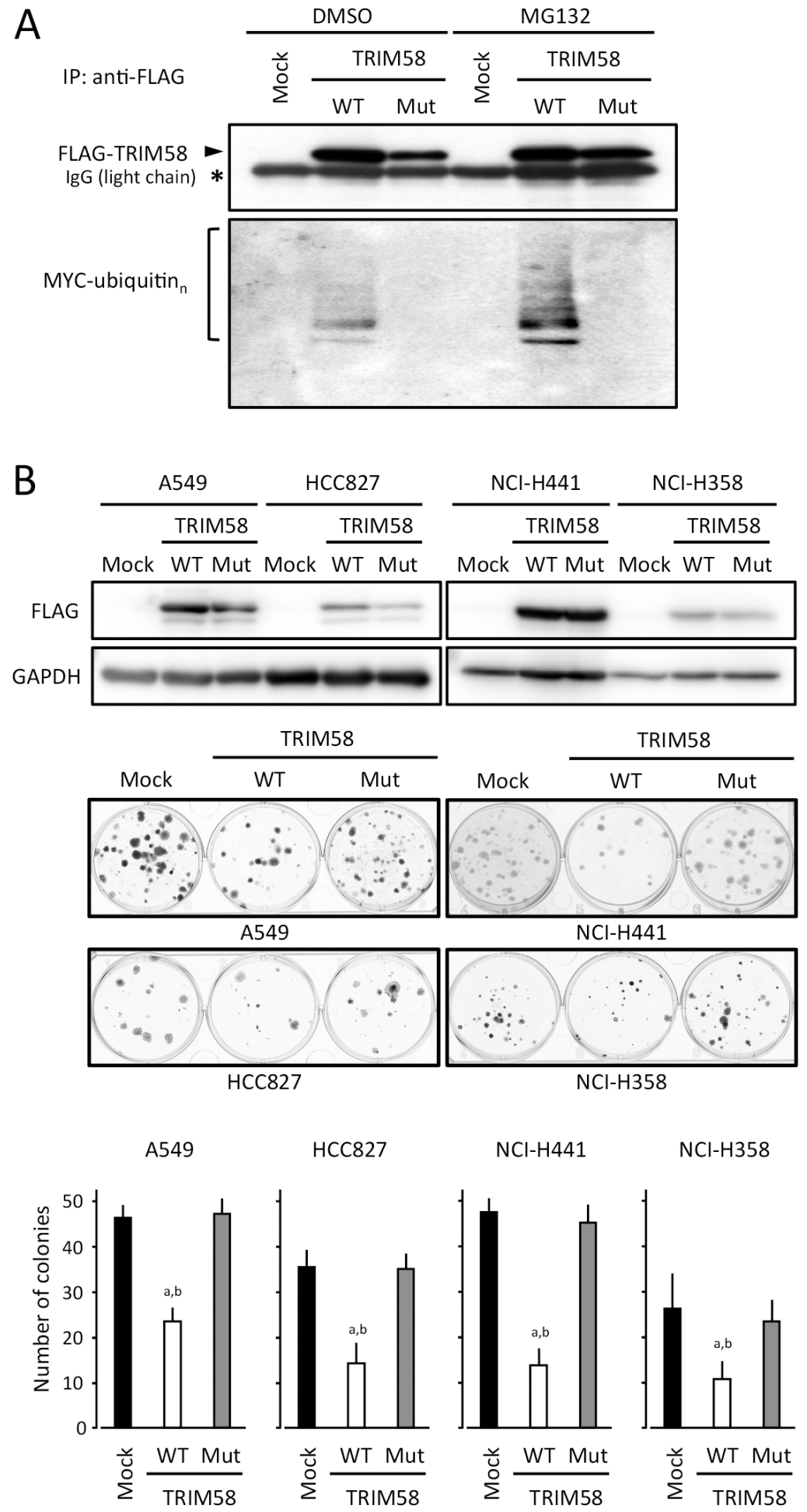

Figure 4: Suppressive effects of transiently transfected TRIM58 on the proliferation of lung adenocarcinoma (LADC) cells in vitro. (A) Ubiquitin activities of wild-type and mutant TRIM58 were determined by detecting the autoubiquitination of TRIM58 proteins exogenously expressed in HEK293 cells. Cells were transiently transfected with MYC-tagged Ub and pCMV-3Tag1A (mock), pCMV-3Tag1A-wild-type TRIM58 (TRIM58-WT), or pCMV-3Tag1A-mutant TRIM58 (TRIM-Mut), cultured for $40 \mathrm{~h}$, and treated with $50 \mu$ M MG132 or vehicle (DMSO) for $8 \mathrm{~h}$. Cell lysates were immunoprecipitated with an anti-FLAG antibody and blotted using antibodies specific for FLAG (upper) or MYC (lower). (B) LADC cell lines transfected with pCMV-3Tag1A (mock), pCMV-3Tag1A-wild-type TRIM58 (TRIM58-WT), or pCMV-3Tag1 A-mutant TRIM58 (TRIM58-Mut) were plated in 6-well plates and treated with $0.5 \mathrm{mg} / \mathrm{mL}$ G418 for two weeks. FLAG-tagged TRIM58 expression was confirmed by western blotting with an anti-FLAG antibody (upper). Drug-resistant colonies were stained with crystal violet and counted (middle). Columns, means \pm standard deviations of four independent experiments (lower). ${ }^{*} P<0.05$ versus mock control, Welch's $t$-test. 
A

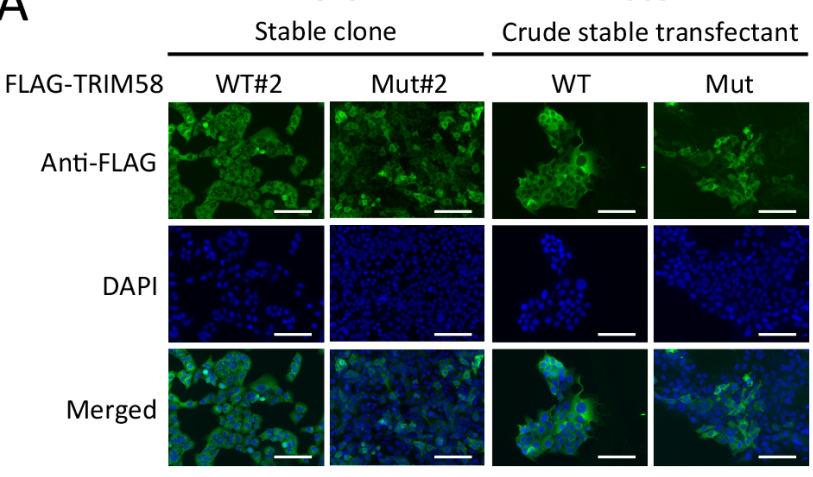

A549

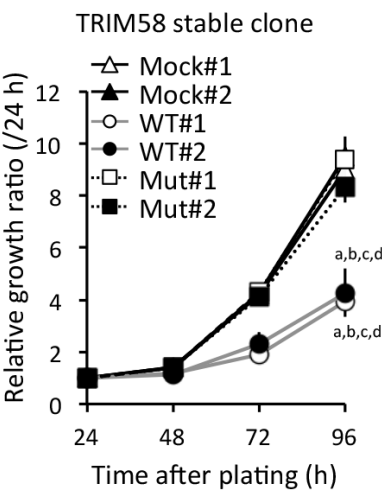

$\mathrm{HCC} 827$

TRIM58

crude stable transfectant

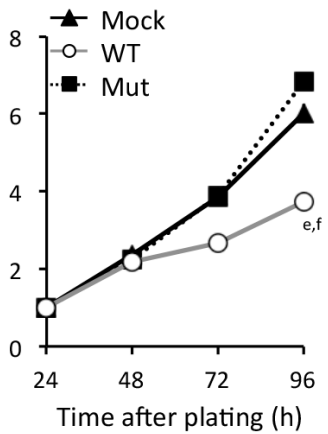

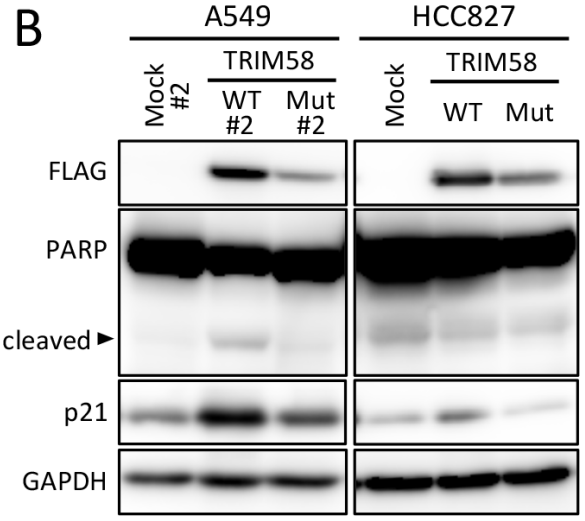

D

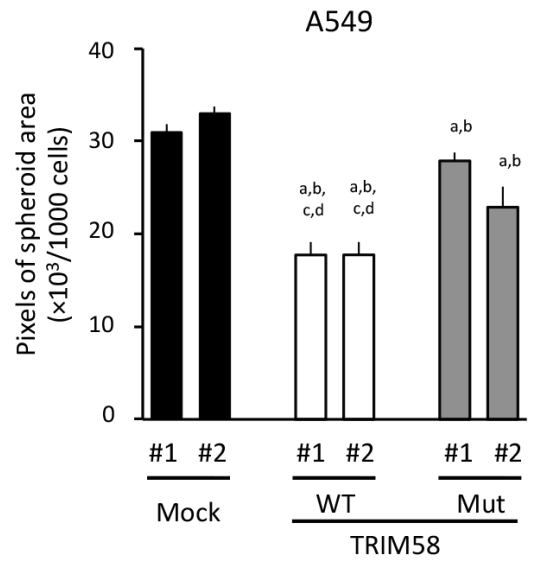

C

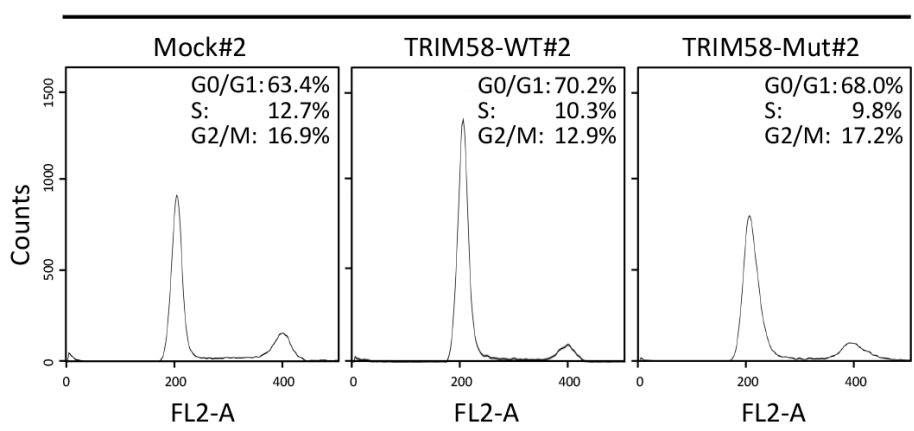

$E$

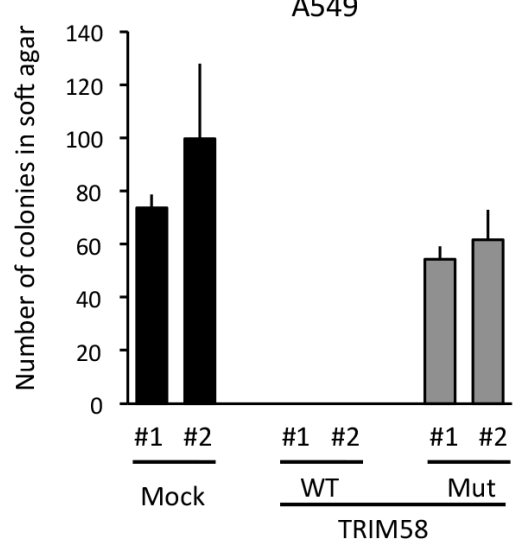

Figure 5: Suppressive effects of stably restored TRIM58 expression on the proliferation of lung adenocarcinoma (LADC) cells in vitro. (A) Left, representative images of A549 or HCC827 cells engineered to stably express FLAG-tagged exogenous wild-type TRIM58 (TRIM58-WT) or mutant TRIM58 (TRIM58-Mut), detected by FIC with an anti-FLAG antibody (green). Nuclei were counterstained with DAPI (blue). Scale bars, $20 \mu \mathrm{m}$. Right, numbers of viable cells for each stable transfectant were assessed using a WST assay for the indicated times. Values are expressed as fold changes (mean \pm standard deviation $[\mathrm{SD}], n=8$ ) relative to the respective control values (24 h). a,b,c,d $P<0.05$ compared with the A549 mock\#1, mock\#2, TRIM58-Mut\#1, and TRIM58-Mut\#2 clones, respectively, at $96 \mathrm{~h}$ after plating. e,f $P<0.05$ compared with the HCC 827 crude mock and TRIM58-Mut transfectants, respectively, at $96 \mathrm{~h}$ after plating. (B) Levels of PARP, p21, and exogenous TRIM58 protein expression in each stable transfectant were analyzed by western blotting, using GAPDH as a loading control. (C)Representative results of the population in each phase of the cell cycle in A549 stable transfectants assessed by a FACS analysis. (D) Spheroid formation assay. A549 stable transfectants were seeded in ultra-low attachment 96-well round bottom plates and incubated at $37^{\circ} \mathrm{C}$ for seven days. Areas of the resulting spheroids were determined as described in the Materials and Methods section (mean $\pm \mathrm{SD}, n=8$ ). a,b,c,d $P<0.05$ compared to A549 mock\#1, mock\#2, TRIM58-Mut\#1, and TRIM58-Mut\#2 clones, respectively. (E) Colony formation assay in soft agar. A549 stable transfectants were cultured in semi-solid medium for 26 days as described in the Materials and Methods section (mean $\pm \mathrm{SD}, n=3$ ). 
Table 3: Partial list of the enriched gene ontology clusters based on the set of differentially expressed genes in TRIM58-overexpressed A549 cells

\begin{tabular}{|c|c|c|c|c|}
\hline Annotation Cluster 1 & Enrichment Score: 7.29 & & & \\
\hline Category & Term & Gene count & $P$-Value ${ }^{\text {a }}$ & Adjusted P-value \\
\hline GOTERM_CC_FAT & extracellular region part & 133 & $1.0 \mathrm{E}-10$ & $4.8 \mathrm{E}-08$ \\
\hline GOTERM_CC_FAT & extracellular region & 224 & $2.4 \mathrm{E}-08$ & $3.8 \mathrm{E}-06$ \\
\hline GOTERM_CC_FAT & extracellular space & 84 & $5.5 \mathrm{E}-05$ & $1.6 \mathrm{E}-03$ \\
\hline Annotation Cluster 2 & Enrichment Score: 7.15 & & & \\
\hline Category & Term & Gene count & $P$-Value ${ }^{\mathrm{a}}$ & Adjusted $P$-value \\
\hline GOTERM_CC_FAT & extracellular region part & 133 & $1.0 \mathrm{E}-10$ & $4.8 \mathrm{E}-08$ \\
\hline GOTERM_CC_FAT & extracellular matrix & 64 & $2.4 \mathrm{E}-10$ & $5.7 \mathrm{E}-08$ \\
\hline SP_PIR_KEYWORDS & extracellular matrix & 47 & $8.1 \mathrm{E}-09$ & $1.7 \mathrm{E}-06$ \\
\hline GOTERM_CC_FAT & proteinaceous extracellular matrix & 56 & $3.1 \mathrm{E}-08$ & $3.7 \mathrm{E}-06$ \\
\hline SP_PIR_KEYWORDS & basement membrane & 14 & $3.9 \mathrm{E}-06$ & $6.0 \mathrm{E}-04$ \\
\hline GOTERM_CC_FAT & extracellular matrix part & 25 & $1.3 \mathrm{E}-05$ & $5.9 \mathrm{E}-04$ \\
\hline GOTERM_CC_FAT & basement membrane & 19 & $2.9 \mathrm{E}-05$ & $1.1 \mathrm{E}-03$ \\
\hline Annotation Cluster 3 & Enrichment Score: 5.58 & & & \\
\hline Category & Term & Gene count & $P$-Value ${ }^{\text {a }}$ & Adjusted $\boldsymbol{P}$-value \\
\hline GOTERM_BP_FAT & biological adhesion & 99 & $6.6 \mathrm{E}-08$ & $8.1 \mathrm{E}-05$ \\
\hline GOTERM_BP_FAT & cell adhesion & 98 & $1.2 \mathrm{E}-07$ & $7.3 \mathrm{E}-05$ \\
\hline SP_PIR_KEYWORDS & cell adhesion & 60 & $5.9 \mathrm{E}-06$ & $6.1 \mathrm{E}-04$ \\
\hline GOTERM_BP_FAT & cell-cell adhesion & 39 & $9.9 \mathrm{E}-04$ & $4.5 \mathrm{E}-02$ \\
\hline
\end{tabular}

a $P$-values were obtained by loading all differentially expressed genes (adjusted $P$-value $<0.05$, upregulated and downregulated genes with $>2$-fold changes) into DAVID Functional Annotation Clustering Tool (https://david.ncifcrf.gov/) to reduce annotation redundancy.

${ }^{\mathrm{b}} P$-values were adjusted with the Benjamini-Hochberg correction.

probes for differentially expressed genes (DEGs) based on the criteria of a 2 -fold change and an adjusted $P$-value $<0.05$; specifically, 1036 and 853 probes were significantly upregulated and downregulated, respectively, in TRIM58WT-overexpressing cells relative to mock control cells. To further identify significantly overrepresented Gene Ontology (GO) terms affected by TRIM58 expression in A549 cells, those DEGs were analyzed using The Database for Annotation, Visualization, and Integrated Discovery (DAVID) v6.7 software [9,10]. Among the DEGs, 1562 genes (796 increased genes and 766 decreased genes) were mapped in the DAVID database. After applying a functional annotation clustering algorithm to reduce annotation redundancy, the GO analysis revealed a highly significant change in the expression of genes encoding proteins involved in the extracellular region (Cluster 1, enrichment score 7.29), extracellular matrix (ECM)/basement membrane (Cluster 2, enrichment score 7.15), and cell adhesion/cell-cell adhesion (Cluster 3, enrichment score 5.58), suggesting that TRIM58 might contribute to cell-cell and/or cell-ECM adhesion (Table 3, Supplementary Table S3).

\section{DISCUSSION}

To our knowledge, this study is the first to demonstrate the functional significance of TRIM58 as a TSG in the context of tumorigenesis. In both our panel of LADC cases and a publicly available data set (TCGA), TRIM58 was consistently silenced via hypermethylation in a tumor-specific manner even in the early stages of tumorigenesis and regardless of smoking status. In addition, the restoration of TRIM58 expression in LADC cells inhibited the growth of LADC cells in vitro and in vivo through its $\mathrm{Ub}$ ligase activity. Although the $\mathrm{Ub}$ ligase target substrates that underlie the tumor suppressive activity of TRIM58 remain unknown, this activity appears to associate with cell-cell and/or cell-ECM adhesion, suggesting that the inactivation of TRIM58 might contribute to the epithelial disintegration observed in early carcinogenesis. Taken together, these findings indicate that TRIM58 likely acts as a TSG in the early stages of lung carcinogenesis, such as initiation or early tumorigenesis regardless of smoking-induced "field defects." Indeed, the TRIM58 CGI methylation levels in non-cancerous lung 
tissues of S and NS were similar ( Figure 2E), suggesting that TRIM58 may be a universal epigenetic early diagnostic marker, rather than a prognostic marker, of LADC, and may also serve as a possible preventive and/or therapeutic target through the epigenetic restoration of its expression. Further analyses of the direct Ub-ligase targets
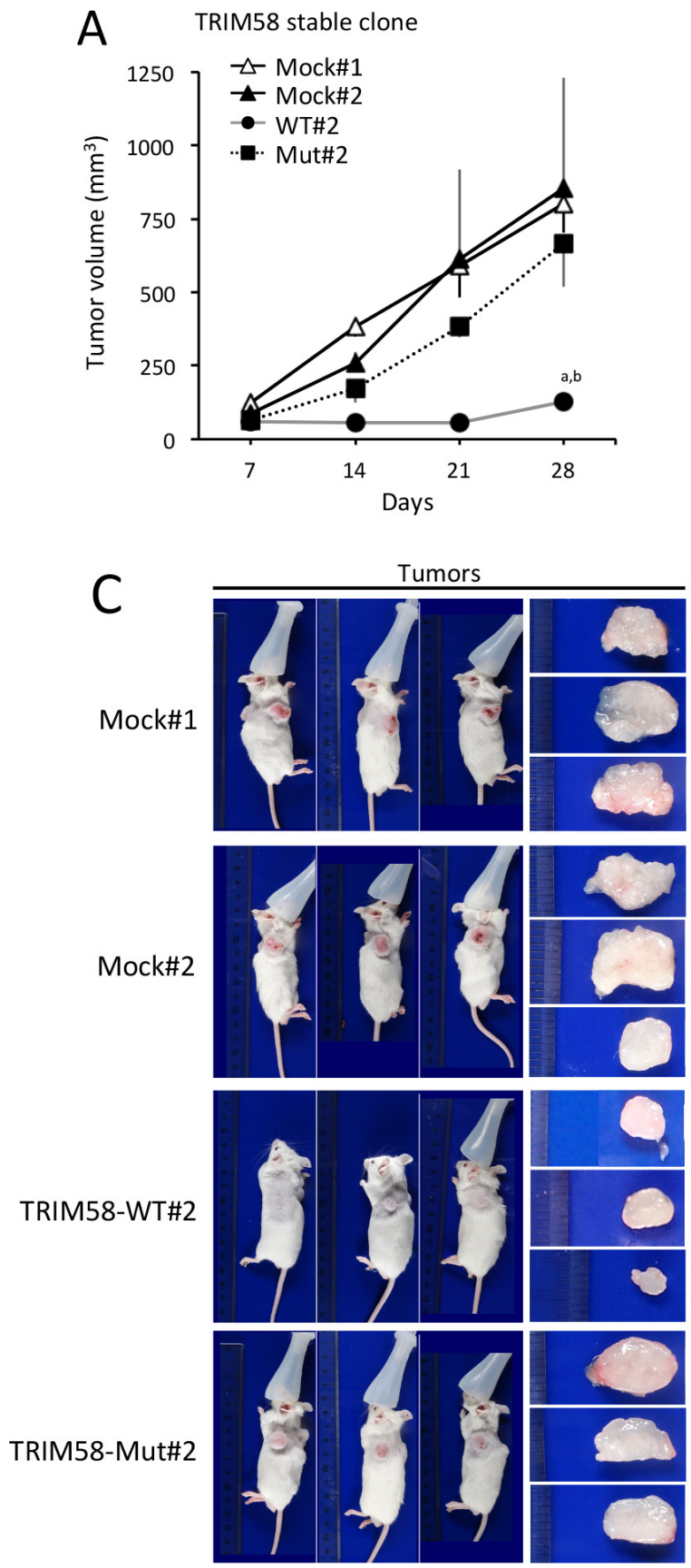

Figure 6: Suppressive effects of restored TRIM58 expression on the formation of A549 cell tumors in vivo. (A) Tumor growth was assessed weekly for up to 28 days after the injection of Matrigel suspensions of each A549 stable transfectant into the flanks of severe combined immunodeficient (SCID) mice. Tumor volumes $\left(\mathrm{mm}^{3}\right)$ were calculated as described in the Materials and Methods section. Time-dependent changes in tumor masses are shown (mean \pm standard deviation [SD], $n=3$ ). ${ }^{\mathrm{a}, \mathrm{b}} P<0.05$ compared to mock\#1 and mock\#2, respectively. (B) The SCID mice used in Figure 6A were sacrificed to evaluate tumor weights on day 28 after inoculation. Mean \pm SD tumor weights are shown $(n=3)$. ${ }^{\mathrm{a}, \mathrm{b}} P<0.05$ compared to mock\#1 and mock\#2, respectively. $(\mathbf{C})$ Representative tumors formed in SCID mice on day 28 after inoculation (left) and immunohistochemical analysis of FLAG-tagged TRIM58 protein (wild-type or mutant) and Ki-67 expression (right). Bars, $100 \mu \mathrm{m}$. 
the findings of a very recent report [11] in which DiazLagares et al. identified TRIM58 as one of four genes that consistently exhibited CGI hypermethylation in non-small cell lung cancers (NSCLCs) relative to non-malignant lung tissues. In addition, Diaz-Lagares and colleagues found that this hypermethylation was associated with transcriptional silencing in early-stage (stage I) NSCLCs, regardless of smoking status, and identified a DNA methylation signature model based on those four genes that yielded high diagnostic accuracy for NSCLCs even in minimally invasive samples, such as bronchial fluids [11]. According to reference databases in which gene expression profiles of normal tissue have been generated using next-generation RNA sequencing technologies, such as RNA-seq Atlas (http://medicalgenomics.org/rna_seq atlas) and RefEx (http://refex.dbcls.jp/), TRIM58 mRNA was not detected in most normal tissues although higher expression of this gene is observed in the lung relative to the levels in various other tissues. Very recently, hypermethylation was found to correlate with decreased TRIM58 expression in hepatocellular carcinoma (HCC) cells relative to paired adjacent liver tissues, although no functional characterization was observed in HCC cells [12]. Given the infrequency of TRIM58 CGI hypermethylation $(28.18 \%, 51 / 181$ cases $)$ and tendency of this event to correlate with unfavorable disease-free survival in HCC [12], hypermethylation appears to occur in more progressive HCC tumors. Therefore, it remains unclear whether TRIM58 plays different tumor suppressive roles in the lung and liver.

The prevalence of $\mathrm{CGI} /$ promoter methylation in lung cancers, including LADC, has been reported to differ between $\mathrm{S}$ and NS; specifically, higher methylation of CDKN2A, MGMT, RASSF1, MTHFR, and FHIT promoters has been observed in $\mathrm{S}$ vs. NS, whereas RASSF2, TNFRSF 10C, BHLHB5, and BOLL methylation is more common in NS than in S [13]. In the present study of LADC, we conducted a genome-wide screen to identify novel driver genes that contribute to early carcinogenesis following aberrant CGI methylation and consequent epigenetic activation or inactivation, regardless of the presence or absence of epigenetic field defects caused by tobacco smoking, in 12 clinically uniform stage-I LADC cases. Of the nine genes clustered around the top 10 hypermethylated CGIs in tumors, several were reported to serve as epigenetic markers of DNA hypermethylation in various malignant diseases, including LADC [9, 14-18]; however, previously described well-known methylation targets in lung cancers [13] were not included in this list. For example, PTPRN2 hypermethylation changes had been previously reported in LADC, even in preneoplastic lesions or atypical adenomatous hyperplasias [14]. Frequent methylation of PTPRN2 and ZNF577 were reported in lung squamous cell carcinomas $[15,16]$. FEZF2 was found to be a 3 p14 TSG that is frequently methylated in nasopharyngeal squamous cell carcinoma
[17]. However, the effects of hypermethylation on the expression of these three genes in LADC cases were small or even opposite to the effects reported in the TCGA data set. In addition, FEZF2 was not expressed in normal lung tissues. Furthermore, silencing of DPP6 through hypermethylation of its promoter was reported in acute myeloid leukemia [18] and an in vitro neuronal differential model [19], whereas hypomethylation and subsequently increased expression of this gene have been found in colon cancer [20], suggesting that the tumor suppressive role of this gene in epithelial tumors remains unknown. Taken together, the biological significance of selected candidate genes as molecular targets for epigenetic modification therapy of LADC remains unclear, with the exception of TRIM58. However, the methylation statuses of these genes, which were validated in the TCGA data set except for MEIS1 and PTPRN2, might be useful as early molecular diagnosis markers of LADC.

\section{MATERIALS AND METHODS}

\section{Primary tissue sample collection}

In total, 76 paired tumor/non-tumorous LADC sample sets were obtained from patients (39 S, $37 \mathrm{NS}$ ) with histologically proven primary LADC who underwent surgery at Tokushima University Hospital (Tokushima, Japan) between April 1999 and March 2015. None of the patients had received preoperative radiation, chemotherapy, or immunotherapy. A total of 12 LADC (6 S and $6 \mathrm{NS}$ ), 46 LADC (25 S and $21 \mathrm{NS}), 38$ LADC (18 S and 20 $\mathrm{NS}$ ), and $63 \mathrm{LADC}$ sets (28 S and $35 \mathrm{NS}$ ) were used for methylation screening with a HumanMethylation450K array, pyrosequencing-based methylation analysis, realtime PCR-based expression analysis, and IHC, respectively (Table 1, Supplementary Table S1).

Tumors were snap-frozen in liquid nitrogen and stored at $-80^{\circ} \mathrm{C}$ until required for DNA and RNA analyses. Tumor specimens were characterized according to the International System of Classification of Tumors, which was based on the tumor-node-metastasis (TNM) classification of the IASLC Lung Cancer Staging Project (7th edition) [21]. Diagnoses were verified by histopathology, and only samples with at least $70 \%$ tumor cells were used in the study.

This study was performed in accordance with the principles outlined in the Declaration of Helsinki. Following the approval of all aspects of these studies by the local ethics committee (Tokushima University Hospital, approval number 2204), formal written consent was obtained from all patients.

\section{DNA and RNA preparation and bisulfite conversion of genomic DNA}

DNA and RNA were extracted using standard methods. Bisulfite conversion of DNA was conducted 
using the EZ DNA Methylation Gold Kit (Zymo Research, Orange, CA, USA).

\section{Global methylation analysis}

The HumanMethylation450K BeadChip (Illumina, Santa Clara, CA, USA) analysis was performed according to the manufacturer's instructions. The default settings of the GenomeStudio Software's DNA methylation module (Illumina) were applied to calculate the methylation levels of $\mathrm{CpG}$ sites as $\beta$-values ( $\beta=$ Intensity (methylated)/ intensity (methylated + unmethylated)). The data were further normalized using a peak correction algorithm embedded in the R-package Illumina Methylation Analyzer (IMA). To identify differentially methylated CGIs between tumors and paired non-tumorous lung tissues in the discovery LADC set, median-averaged $\beta$-differences in CGI-based regions were calculated based on a $\beta$-differences matrix in which $\beta$-values of paired non-tumorous lung tissues were subtracted from those of tumors; the statistical significance of these differences was evaluated using Welch's $t$-test in IMA. Multiple testing corrections were performed using a Benjamini-Hochberg approach with significantly differential methylation, defined as a FDRadjusted $P$-value $<0.05$. The following criteria were used for differentially methylated CGIs: a $\beta$-difference $>0.25$ and FDR-adjusted $P$-value $<0.05$. Methylation data for the discovery cohort were deposited in the Gene Ontology Database (GEO) under accession number GSE83842.

\section{Cell lines}

A total of 14 LADC cell lines (A549, ABC1, HCC827, NCI-H358, NCI-H441, NCI-H522, NCI-H1437, NCI-H1650, NCI-H1975, PC3, PC14, RERF-LC-KJ, RERF-LC-MS, VMRC-LCD) were obtained from the Japanese Collection of Research Bioresources (Ibaraki, Japan), RIKEN BioResource Center (Tsukuba, Japan), or American Type Culture Collection (Manassas, VA, USA) and cultured in appropriate media. To analyze the effects of DNA demethylation and/or histone acetylation on gene expression, cells were treated with $1 \mu \mathrm{M} 5$-aza-dC for five days and/or $300 \mathrm{nM}$ trichostatin A (TSA) for the last $12 \mathrm{~h}$ of culture.

\section{Primers}

Primers used in this study are listed in Supplementary Table S4.

\section{Quantitative real-time PCR (qRT-PCR)}

qRT-PCR was performed using specific primer sets (Supplementary Table S4) and SYBR Green Master Mix (Applied Biosystems, Waltham, MA, USA) as described elsewhere [22], or TaqMan kits (Supplementary
Table S4) according to the manufacturer's instructions. $G A P D H$ mRNA levels were used as internal controls for normalization.

\section{Processing of the TCGA data set}

Available RNA-seq data (IlluminaHiSeq_RNASeqV2 Level 3) and Infinium HumanMethylation450K data (Level 1) for clinical LADC specimens with clinical annotations were downloaded from TCGA Research Network (http://cancergenome.nih.gov). Gene expression data and DNA methylation data were available for 58 and 29 paired tumor/non-tumorous LADC sample sets, respectively; both types of data without outliers were available for 17 sets. The DESeq2 package was applied to raw RSEM data to perform data normalization and evaluate the differential expression status of each transcript between tumors and non-tumorous tissues [23].

To evaluate CGI methylation statuses, IDAT files were preprocessed using the R-package IMA, and the methylation level of each CG site was calculated as described above. We computed the differential gene expression and methylation statuses between tumors and paired non-tumorous tissues and evaluated statistical significance using a paired $t$-test with multiple testing corrections via the BenjaminiHochberg approach (FDR-adjusted $P$-value).

\section{Antibodies}

Antibodies used in this study are listed in Supplementary Table S5.

\section{Western blot analysis}

Whole-cell lysate preparation and western blot analysis were performed as previously described [22].

\section{Bisulfite pyrosequencing and bisulfite genomic sequencing (BGS)}

Bisulfite-treated genomic DNA was amplified using a set of primers designed with PyroMark Assay Design Software (version 2.0.01.15; Qiagen, Valencia, CA, USA; Supplementary Table S4). PCR product pyrosequencing and methylation quantification were performed on a PyroMark 24 Pyrosequencing System, version 2.0.6 (Qiagen) with sequencing primers according to the manufacturer's instructions. For BGS, bisulfite-treated genomic DNA were amplified, subcloned, and sequenced as described previously [5].

\section{Promoter reporter assay}

Six DNA fragments around the TRIM58 CGI were obtained via PCR with specific primer sets (Supplementary Table S4) and subsequently ligated into the pGL3-Basic 
vector (Promega, Madison, WI, USA). A promoter reporter assay was performed using reporter constructs as described previously [5].

\section{Immunohistochemical staining}

Paraffin-embedded sections (thickness, $4 \mu \mathrm{m}$ ) were subjected to IHC staining using specific antibodies for each protein (Supplementary Table S5) and the Envision system (ChemMate Envision kit; Dako, Glostrup, Denmark) according to the manufacturer's instructions.. Antigen retrieval was performed by heating dewaxed and dehydrated sections in Dako Real Target Retrieval Solution, pH 9 (DAKO, Glostrup, Denmark) at $98^{\circ} \mathrm{C}$ for $30 \mathrm{~min}$.

\section{Fluorescent immunocytochemical staining}

Cells cultured on chamber slides were fixed, permeabilized, and stained using specific primary antibodies and fluorescently labeled secondary antibodies (Supplementary Table S5) as described elsewhere [22]. Stained cells were observed under a fluorescence microscope (LSM510; Carl Zeiss, Oberkochen, Germany).

\section{Expression plasmid construction}

The full coding sequence of human TRIM58 (NM_015431) was amplified via PCR (Supplementary Table S4) using cDNA prepared from normal lung RNA (Takara, Shiga, Japan). This coding sequence was then cloned into the mammalian expression vector pCMV-3Tag1A (Stratagene, La Jolla, CA, USA) with the intent to append the FLAG epitope to the $\mathrm{NH}_{2}$ terminus of TRIM58. The product was amplified by PCR (Supplementary Table S4) and cloned into the retroviral vector pMXs-Neo (Cell Biolabs, San Diego, CA, USA). pTRIM58-WT-FLAG harboring the H33A mutation (pTRIM58-Mut-FLAG) was generated via site-directed mutagenesis (Supplementary Table S4) with a KOD -plus- Mutagenesis Kit (TOYOBO, Osaka, Japan).

\section{Transient transfection experiments}

A control plasmid (pCMV-3Tag1A), pTRIM58WT-FLAG, and pTRIM58-Mut-FLAG were transfected separately into LADC cell lines or HEK293 cells using Lipofectamine 2000 reagent (Invitrogen, Carlsbad, CA, USA).

\section{Ubiquitin assay}

HEK293T cells at $<80 \%$ confluence were cotransfected with a MYC-tagged ubiquitin-expressing plasmid and either a control plasmid (pCMV-3Tag1A), pTRIM58-WT-FLAG, or pTRIM58-Mut-FLAG for $48 \mathrm{~h}$. Cells were detached by scraping in $500 \mu \mathrm{L}$ of RIPA lysis buffer and lysed on ice for $10 \mathrm{~min}$. The lysates were then centrifuged at $14,000 \mathrm{rpm}$ and $4^{\circ} \mathrm{C}$ for $10 \mathrm{~min}$, after which the supernatants (40 $\mu \mathrm{g}$ protein) were incubated for $1 \mathrm{~h}$ at room temperature with $20 \mu \mathrm{L}$ of a $50 \%(\mathrm{v} / \mathrm{v})$ suspension of Anti-FLAG M2 Agarose Affinity Gel (Sigma, St. Louis, MO, USA). Proteins bound to the gel were pulled down and assayed by western blotting with an anti-MYC antibody, as described above.

\section{Stable transfection experiments}

To establish LADC cell lines that would stably overexpress wild-type or mutant TRIM58 protein, cells were infected with each TRIM58-expressing retrovirus and selected by treatment with $0.5 \mathrm{mg} / \mathrm{mL}$ G418 for four weeks. Control cells were obtained using retroviruses generated from an empty pMXs-Neo vector (mock) and packaged in PLAT-A cells. Clones were subsequently isolated, subcultured, and tested for TRIM58 overexpression by western blotting and FIC.

\section{Cell proliferation and cell cycle analyses}

For colony formation assays, cells were transiently transfected with either mock-, pTRIM58-WT-FLAG-, or pTRIM58-Mut-FLAG-expressing plasmids (1,000 cells/ well), plated in 6-well plates, and treated with $0.5 \mathrm{mg} / \mathrm{mL}$ G418 for two weeks. The expression of each TRIM58 protein variant was confirmed $48 \mathrm{~h}$ after transfection by western blotting. The colonies in each well were stained with crystal violet to allow quantification.

Cell growth was assessed at the indicated time after cell seeding $\left(1 \times 10^{4}\right.$ cells/96-well plate $)$ using a watersoluble tetrazolium (WST) salt assay (Cell Counting Kit-8; Dojindo, Mashikimachi, Japan) according to the manufacturer's instructions. The results are expressed as the mean absolute absorbance at the indicated time divided by the mean absolute absorbance of each sample cultured for $24 \mathrm{~h}$ after seeding. Cell cycle was evaluated by FACS as described elsewhere [22]. Raw cell cycle analysis data were quantified using FlowJo software (Treestar, Ashland, OR, USA).

Spheroid formation assays were performed according to a previously detailed description [22]. The areas of the resulting spheroids were determined using ImageJ software (National Institutes of Health, Bethesda, MD, USA; http://imagej.nih.gov/ij/). For soft-agar colony formation assays, a $60-\mathrm{mm}$ diameter culture dish was coated with $3 \mathrm{ml}$ of bottom agarose mixture (RPMI$1640 / 10 \% \mathrm{FBS} / 0.5 \%$ agarose). After the bottom layer had solidified, $2 \mathrm{ml}$ of top agarose mixture (RPMI-1640/10\% FBS $/ 0.33 \%$ agarose) containing $1 \times 10^{5}$ cells was added to each well. Triplicate cultures were incubated in a $5 \%$ $\mathrm{CO}^{2} / 95 \% \mathrm{O}^{2}$ atmosphere at $37^{\circ} \mathrm{C}$. At 26 days after plating, the colonies were stained with $0.03 \%$ crystal violet, photographed, and counted. 


\section{Animal studies}

The experimental use of animals described herein was approved by the Animal Care Committee of Tokushima University (approval number 13105). Five-week-old male severe combined immunodeficient (SCID) mice (CLEA Japan, Tokyo, Japan) were caged in groups of six and acclimated for one week. A suspension of cells from each stably transfected clone $\left(2 \times 10^{6}\right.$ cells $)$ was prepared in Matrigel (Corning, Corning, NY, USA) and injected subcutaneously into the bilateral flanks of SCID mice. Resulting tumor sizes were measured in two dimensions with a caliper, and volumes were calculated using the following formula: (short diameter) $)^{2} \times$ (long diameter)/2. Recipient mice were sacrificed for tumor weight evaluation and protein expression analyses four weeks after tumor injection, and the growing tumors were removed and fixed with formalin in phosphate-buffered saline. Fixed xenografts were sectioned and subjected to IHC with antibodies specific for FLAG or Ki-67 (Supplementary Table S5).

\section{Expression array analysis and GO and pathway analyses of selected genes}

LADC cell lines engineered to stably overexpress TRIM58-WT and its control counterparts were used in an mRNA analysis with a SuperPrint G3 Human GE $8 \times 60 \mathrm{k}$ Microarray (Agilent Technologies, Santa Clara, CA, USA). RNA integrity was assessed using an Agilent Bioanalyzer 2100 (Agilent Technologies). Sample preparation, slide hybridization, and analysis were performed according to the manufacturer's standard protocols. Data were analyzed using Gene Spring GX13.0 (Agilent Technologies) and deposited to the GEO under accession number GSE85301. After applying a Benjamini-Hochberg multiple testing correction, genes expressed differentially between groups were identified as those with adjusted $P$-values $<0.05$. A gene set comprising upregulated and downregulated genes with $>2$-fold changes in TRIM58-WT-expressing A549 cells relative to control counterparts was analyzed using a standard enrichment analysis (DAVID Bioinformatics Resources 6.7, https://david.ncifcrf.gov/). The enrichment $P$-value calculation was performed using a BenjaminiHochberg multiple testing correction.

\section{Statistical analysis}

Results are expressed as means \pm standard deviations (SDs). Welch's $t$-test or the Mann-Whitney $U$ test was used for comparisons between two groups. The paired $t$-test and Wilcoxon signed-rank test were used for comparisons between paired samples when the data were and were not normally distributed, respectively. Multiple group comparisons were conducted using a one-way analysis of variance followed by Dunnett's post-hoc test or a Kruskal-Wallis analysis of variance followed by the Steel-Dwass post-hoc test. The relation between continuous variables was investigated by means of Pearson's correlation coefficient. Differences were assessed with a two-sided test and considered significant at a $P$-value of $<0.05$. Statistical analyses were performed using R 3.2.2 (R Project for Statistical Computing, Vienna, Austria).

\section{CONFLICTS OF INTEREST}

The authors have no conflicts of interest to declare.

\section{GRANT SUPPORT}

This study was supported in part by JSPS KAKENHI Grant Numbers 26293304 (I.I.), 16K15618 (I.I.), 24590943 (K.M), $15 \mathrm{~K} 19620$ (T.N.) and 25861245(K.K.).

\section{REFERENCES}

1. Torre LA, Bray F, Siegel RL, Ferlay J, Lortet-Tieulent J, Jemal A. Global cancer statistics, 2012. Cancer J Clin. 2015; 65:87-108.

2. Travis WD, Brambilla E, Noguchi M, Nicholson AG, Geisinger KR, Yatabe Y, Beer DG, Powell CA, Riely GJ, Van Schil PE, Garg K, Austin JH, Asamura H, et al. International association for the study of lung cancer/ american thoracic society/european respiratory society international multidisciplinary classification of lung adenocarcinoma. J Thorac Oncol. 2011; 6:244-285.

3. Johnson DH, Schiller JH. Recent clinical advances in lung cancer management. J Clin Oncol 2014; 32:973-982.

4. Izumi $\mathrm{H}$, Inoue $\mathrm{J}$, Yokoi $\mathrm{S}$, Hosoda $\mathrm{H}$, Shibata $\mathrm{T}$, Sunamori M, Hirohashi S, Inazawa J, Imoto I. Frequent silencing of $\mathrm{DBC} 1$ is by genetic or epigenetic mechanisms in non-small cell lung cancers. Hum Mol Genet. 2005; 14:997-1007.

5. Imoto I, Izumi H, Yokoi S, Hosoda H, Shibata T, Hosoda F, Ohki M, Hirohashi S, Inazawa J. Frequent silencing of the candidate tumor suppressor PCDH20 by epigenetic mechanism in non-small-cell lung cancers. Cancer Res. 2006; 66:4617-4626.

6. Toyooka S, Tokumo M, Shigematsu H, Matsuo K, Asano H, Tomii K, Ichihara S, Suzuki M, Aoe M, Date H, Gazdar AF, Shimizu N. Mutational and epigenetic evidence for independent pathways for lung adenocarcinomas arising in smokers and never smokers. Cancer Res. 2006; 66:1371-1375.

7. Tessema M, Yingling CM, Liu Y, Tellez CS, Van Neste L, Baylin SS, Belinsky SA. Genome-wide unmasking of epigenetically silenced genes in lung adenocarcinoma from smokers and never smokers. Carcinogenesis. 2014; $35: 1248-1257$. 
8. Thom CS, Traxler EA, Khandros E, Nickas JM, Zhou OY, Lazarus JE, Silva AP, Prabhu D, Yao Y, Aribeana C, Fuchs SY, Mackay JP, Holzbaur EL, et al. Trim58 degrades Dynein and regulates terminal erythropoiesis. Dev Cell. 2014; 30:688-700.

9. Huang W, Sherman BT, Lempicki RA. Systematic and integrative analysis of large gene lists using DAVID bioinformatics resources. Nat Protoc. 2009; 4:44-57.

10. Huang DW, Sherman BT, Lempicki RA. Bioinformatics enrichment tools: paths toward the comprehensive functional analysis of large gene lists. Nucleic Acids Res. 2009; 37:1-13.

11. Diaz-Lagares A, Mendez-Gonzalez J, Hervas D, Saigi M, Pajares MJ, Garcia D, Crujeiras AB, Pio R, Montuenga LM, Zulueta J, Nadal E, Rosell A, Esteller M, et al. A novel epigenetic signature for early diagnosis in lung cancer. Clin Cancer Res. 2016; 22:3361-3371.

12. Qiu X, Huang Y, Zhou Y, Zheng F. Aberrant methylation of TRIM58 in hepatocellular carcinoma and its potential clinical implication. Oncol Rep. 2016; 36: 811-818.

13. Larsen JE, Minna JD. Molecular biology of lung cancer: clinical implications. Clin Chest Med. 2011; 32:703-740.

14. Selamat SA, Galler JS, Joshi AD, Fyfe MN, Campan M, Siegmund KD, Kerr KM, Laird-Offringa IA. DNA methylation changes in atypical adenomatous hyperplasia, adenocarcinoma in situ, and lung adenocarcinoma. PLoS ONE. 2011; 6:e21443.

15. Anglim PP, Galler JS, Koss MN, Hagen JA, Turla S, Campan M, Weisenberger DJ, Laird PW, Siegmund KD, Laird-Offringa IA. Identification of a panel of sensitive and specific DNA methylation markers for squamous cell lung cancer. Mol Cancer. 2008; 7:62.

16. Rauch TA, Wang Z, Wu X, Kemstine KH, Riggs AD, Pfeifer GP. DNA methylation biomarkers for lung cancer. Tumour Biol. 2012; 33:287-296.
17. Shu XS, Li L, Ji M, Cheng Y, Ying J, Fan Y, Zhong L, Liu X, Tsao SW, Chan AT, Tao Q. FEZF2, a novel 3p14 tumor suppressor gene, represses oncogene EZH2 and MDM2 expression and is frequently methylated in nasopharyngeal carcinoma. Carcinogenesis. 2013; 34:1984-1993.

18. Saied MH, Marzec J, Khalid S, Smith P, Down TA, Rakyan VK, Molloy G, Raghavan M, Debernardi S, Young BD. Genome wide analysis of acute myeloid leukemia reveal leukemia specific methylome and subtype specific hypomethylation of repeats. PLoS One. 2012; 7:e33213.

19. Sheikh MA, Malik YS, Yu H, Lai M, Wang X, Zhu X. Epigenetic Regulation of Dpp6 Expression by Dnmt3b and Its Novel Role in the Inhibition of RA Induced Neuronal Differentiation of P19 Cells. PLoS One. 2013; 8:e55826

20. Irizarry RA1, Ladd-Acosta C, Wen B, Wu Z, Montano C, Onyango P, Cui H, Gabo K, Rongione M, Webster M, Ji H, Potash JB, Sabunciyan S, et al. The human colon cancer methylome shows similar hypo- and hypermethylation at conserved tissue-specific $\mathrm{CpG}$ island shores. Nat Genet. 2009; 41:178-186.

21. Detterbeck FC, Boffa DJ, Tanoue LT. The new lung cancer staging system. Chest. 2009; 136:260-271.

22. Hamada J, Shoda K, Masuda K, Fujita Y, Naruto T, Kohmoto T, Miyakami Y, Watanabe M, Kudo Y, Fujiwara H, Ichikawa D, Otsuji E, Imoto I. Tumor-promoting function and prognostic significance of the RNA-binding protein T-cell intracellular antigen-1 in esophageal squamous cell carcinoma. Oncotarget. 2016; 7:17111-17128. doi: 10.18632/oncotarget.7937.

23. Love MI, Huber W, Anders S. Moderated estimation of fold change and dispersion for RNA-seq data with DESeq2. Genome Biology. 2014; 15:550. 